\title{
PREVALÊNCIA DE GESTAÇÃO ECTÓPICA DE TRATAMENTO CIRÚRGICO EM HOSPITAL PÚBLICO DE 1995-2000
}

\author{
*Arlete Maria dos Santos Fernandes, luciana Peres Ribeiro, Flávia Helena Moraes, \\ Poliana Cunha Meira, Celina de Azevedo Sollero, Elza Mitiko Yamada \\ Trabalho realizado no Departamento de Tocoginecologia, Faculdade de Ciências Médicas e Área \\ de Tocoginecologia, Faculdade de Ciências Médicas, Pontifícia Universidade Católica de Campinas, SP
}

RESUMO - Objetivos. Determinar a prevalência de gestação ectópica (GE) em mulheres submetidas à laparotomia no Hospital e Maternidade Celso Pierro durante os anos de 1995 a 2000, avaliar as características e o tratamento realizado.

Métodos. Estudo retrospectivo. Os prontuários de mulheres submetidas à laparotomia foram revisados. As variáveis consideradas foram idade, procedência da mulher, trabalho, paridade, gravidez ectópica íntegra ou rôta, cirurgia realizada, complicações e tempo de internação. A análise de dados foi descritiva, seguida de regressão logística.

RESULTADOs. Noventa e seis casos foram identificados, todos com GE tubária. A maioria das mulheres (65\%) tinha entre 25 e 35 anos e $30 \%$ estavam empregadas. A maior parte dos diagnósticos foi de gestação ectópica rôta (66\%) e realizou-se salpingectomia com ou sem ooforectomia em $79 \%$. Houve $19 \%$ de casos complicados, com maior freqüência de anemia aguda, um óbito por CIVD e 12\% das mulheres receberam transfusão. 0 tempo médio de internação foi de 3,63 dias $(\mathrm{DP} \pm 0,8)$. A prevalência de gestações ectópicas manteve-se estável durante o período e correspondeu a II\% do total de laparotomias.

ConclusöEs. 0 número de GE submetidas à cirurgia manteve-se estável durante o período estudado, 0 que requer medidas preventivas em vários níveis para o seu controle. Esta é patologia grave, cursa com alta morbidade e segue sendo desafio nos serviços de ginecologia.

UnIteRMOs: Gestação ectópica. Hospitalização. Prevalência.

\section{INTRODUÇÃO}

A incidência da gestação ectópica (GE) tem aumentado nas últimas décadas devido a vários fatores. Por um lado, a dosagem da subunidade beta da gonadotrofina coriônica, os ecógrafos de maior precisão e o uso da endoscopia ginecológica facilitaram o diagnóstico. Por outro, a disseminação das infecções genitais por clamídia e gonococos têm elevado o número de mulheres com seqüela tubária, o que também contribui para o aumento das gestações ectópicas'. Apesar da mortalidade ter diminuído ao longo da última década, através do diagnóstico precoce, ainda a gravidez ectópica tem sido responsabilizada por $9 \%$ das mortes no primeiro trimestre da gestação nos Estados Unidos'. No Brasil, a mortalidade por gestação ectópica insere-se entre as causas hemorrágicas. Segundo dados do Estado de São Paulo entre os anos de 199| - 1995, a taxa de mortalidade materna devido a todas as causas variou de 43,7 a 49,6 por 100.000 nascidos vivos ${ }^{2}$.

\section{* Correspondência \\ Caixa postal 6181}

CEP 13084-971 - Campinas - SP - Tel: (19) 3289-2856 Fax: (19) 3289-2440 -E-mail: arlete@fcm.unicamp.br
Vários fatores se associam ao aumento de risco para GE durante a vida reprodutiva. Dentre eles são citados o uso de DIU (RR I I,9) e de citrato de clomifeno (RR I0,0), antecedente de cirurgia tubária (RR 5,6) e de doença inflamatória pélvica (RR 4,0$)$ entre os que mais contribuem para o risco relativo ${ }^{3}$. Outros fatores são citados, como infertilidade, aborto induzido, aderências pélvicas, cirurgias abdominais, malformações uterinas, miomas e contraceptivos de progestágenos ${ }^{3}$. Em estudo recente com populações européias, entre os mais importantes fatores de risco para GE foram citados $\mathrm{O}$ antecedente de doença pélvica infecciosa e o hábito de fumar, e contribuíram outros fatores como a infertilidade, abortos espontâneos e uso de DIU4. A gestação ectópica é considerada uma das complicações possíveis após infecção de trato reprodutivo ${ }^{5}$

A doença inflamatória pélvica (DIP) é fator de risco importante na população e constitui complicação de uma DST. Após a infecção do trato genital inferior feminino pela Neisseria gonorrhoeae e/ou Chlamydia thrachomatis existe inicialmente quadro de cervicite, diagnosticada pela presença de secreção mucopurulenta pelo orifício externo do colo uterino, juntamente com processo inflamatório local, podendo ser acompanhado por dor pélvica crônica ou dispareunia. A ascensão do processo infeccioso do cérvice ao aparelho reprodutivo alto, causando salpingite, hidrossalpinge e abscesso ovariano, denomina-se DIP. Essa evolução é insidiosa, na maior parte das vezes pouco ou nada sintomática, fazendo com que poucas mulheres busquem tratamento. $O$ conseqüente dano parcial ou completo das tubas de Falópio permite que essas mulheres, no futuro, vivenciem como seqüela gravidezes ectópicas, esterilidade definitiva e/ou dor pélvica crônica. $O$ controle da infecção, tanto por sua prevalência quanto devido ao alto número de casos assintomáticos, tem custo alto ${ }^{6}$.

No Brasil, não temos medidas de prevalência da DIP, nem de suas seqüelas, sendo a gestação ectópica uma delas. Apesar da ocorrência de GE poder limitar o futuro reprodutivo da mulher ${ }^{7}$, o diagnóstico precoce leva a tratamentos minimamente invasivos, muitas vezes clínico ou cirúrgico conservador, com menor número de complicações e menor morbimor- 
Fernandes AMS et AL.

talidade. Para o tratamento clínico, a administração de metotrexate tem tido excelentes resultados ${ }^{8}$, entretanto, é preconizado para casos onde o saco gestacional íntegro possui tamanho de até $3 \mathrm{~cm}$ e os batimentos do embrião estão ausentes, ou seja, nos casos de diagnóstico precoce?. A laparoscopia é procedimento de escolha para o tratamento, mas em muitos de nossos serviços existe dificuldade para realizá-la prontamente em todos os casos, além do que, nos quadros tardios, existe potencial dificuldade técnica para realizá-la.

Embora esperado que o diagnóstico seja sempre precoce e o tratamento possa ser clínico ou laparoscópico, ainda muitas muIheres chegam aos hospitais com comprometimento hemodinâmico ou com saco gestacional de maior tamanho, em que a cirurgia laparotômica se impõe, seja por limitação da equipe ou do instrumental endoscópico. $\bigcirc$ atraso no diagnóstico, além de demandar o tratamento cirúrgico, na maioria das vezes com amputação da tuba ou do anexo, aumenta o período de internação e as complicações elevam a morbidade e a mortalidade.

Os objetivos deste estudo foram determinar a prevalência de mulheres com gestação ectópica submetidas a tratamento por laparotomia no serviço de ginecologia do Hospital e Maternidade Celso Pierro, hospital universitário da PUC-Campinas, no período de janeiro de 1995 a dezembro de 2000, avaliar suas características e o tratamento realizado.

\section{Métodos}

Foi um estudo de coorte retrospectivo. $\bigcirc$ número de mulheres submetidas à laparotomia no período de 01/01/1995 a 31//2/2000 e seus prontuários foram localizados através do registro de anestesias e posteriormente revisados. As variáveis estudadas foram idade e procedência da muIher, trabalho fora ou no lar, número de gestações anteriores, diagnóstico de gravidez ectópica íntegra ou rôta, cirurgia realizada, complicações e tempo de internação. A análise de dados foi inicialmente descritiva, mediu-se a freqüência anual da patologia, o número total de laparotomias em mulheres e o número de nascidos vivos no período no mesmo serviço, estabelecendo-se a percentagem anual segundo

\begin{tabular}{ccc}
\hline \multicolumn{4}{c}{ Tabela I - Distribuição das faixas de idade das mulheres submetidas à laparotomia por gravidez ectópica } \\
\hline Faixas de idade & $\mathbf{N}$ & $\%$ \\
$\leq 19$ anos & 6 & 6,2 \\
$20-24$ & 11 & 11,4 \\
$25-29$ & 31 & 32,3 \\
$30-34$ & 32 & 33,3 \\
$\geq 35$ anos & 16 & 16,7 \\
Total & 96 & 100,0 \\
\hline
\end{tabular}

Tabela 2 - Distribuição das mulheres com diagnóstico de GE segundo cirurgia realizada

\begin{tabular}{lcc} 
Cirurgia & $\mathbf{N}^{*}$ & $\%$ \\
\cline { 2 - 3 } Salpingectomia & 70 & 72,9 \\
Conservadora & 20 & 20,8 \\
Anexectomia & 6 & 6,3 \\
Total & 96 & 100,0
\end{tabular}

* 64 mulheres tiveram ectópica rôta e 32 tiveram ectópica íntegra

Tabela 3 - Distribuição das mulheres com gestação ectópica segundo tipo de complicação, tempo de internação e desvio padrão em dias

\begin{tabular}{lcc}
\hline & \multicolumn{2}{c}{ Mulheres } \\
\cline { 2 - 3 } $\begin{array}{l}\text { Complicaçōes } \\
\text { (média em dias) }\end{array}$ & $\mathbf{N}$ & Tempo de internação \\
\cline { 2 - 3 } Semcomplicaçōes & 78 & $3,63(0,81)$ \\
Transfusōes & 12 & $4,27(1,49)$ \\
Lesão alça intestinal & 3 & 6,00 \\
Traqueostomia & 1 & 3,00 \\
Hemoperitônio & $\mid$ & 8,00 \\
CIVD + óbito & $\mid$ & 14,00 \\
Total & 96 & $3,93(0,50)$ \\
\hline
\end{tabular}

cada denominador. Posteriormente, realizou-se regressão logística. Foram utilizados o Excel e o pacote estatístico SAS 8,2.

O estudo teve a aprovação do Comitê de Ética em Pesquisa da FCM-PUC-Campinas.

\section{Resultados}

Foram submetidas à laparotomia 96 mulheres com GE, todas de localização tubária. Do total, 82 delas residiam na cidade e I I procediam de cidades da região metropolitana, somente três mulheres viviam fora da área metropolitana ou em outros Estados. A maior parte das mulheres tinha entre 25 e 35 anos de idade e seis eram adolescentes (Tabela I). Mais da metade das mulheres referiu ser do lar e um terço delas trabalhava fora do lar. Quanto aos antecedentes obstétricos, 82 mulheres $(85,4 \%)$ tinham pelo menos um filho vivo e I 4 ( | 4,6\%) eram nulíparas.
O diagnóstico intra-operatório em dois terços das mulheres $(66,7 \%)$ foi de gestação ectópica rôta e o tratamento realizado foi salpingectomia com ou sem ooforectomia em 79,2\% delas (Tabela 2). Do total de mulheres estudadas, 19\% apresentaram alguma complicação, sendo a mais freqüente a anemia aguda com necessidade de transfusão em $12,5 \%$ dos casos. Houve uma morte por coagulação intravascular disseminada, evoluindo para falência de múltiplos órgãos no $14^{\circ}$ dia de internação (Tabela 3). O tempo médio de internação foi de 3,6 dias (DP $\pm 0,8)$ para as cirurgias sem complicação, variando até 14 dias naquelas com complicações (Tabela 3).

A prevalência de GE submetidas à laparotomia no serviço manteve-se estável durante os seis anos levantados, e correspondeu a II,2\% do total de laparotomias realizadas em mulheres no período estudado (Tabela 4). 
Tabela 4 - Número de gestaçóes ectópicas submetidas à laparotomia, número de laparotomias e número de nascidos no período de 1995-2000

\begin{tabular}{|c|c|c|c|}
\hline Ano & $\begin{array}{c}\text { Número de } \\
\text { gestações ectópicas }\end{array}$ & $\begin{array}{c}\text { Número de } \\
\text { laparotomias } \\
\text { em mulheres }(\% \mathrm{GE})\end{array}$ & $\begin{array}{c}\text { Número de } \\
\text { nascidos vivos (\%GE) }\end{array}$ \\
\hline 1995 & 14 & $142(9,85)$ & $2656(0,52)$ \\
\hline 1996 & 14 & $147(9,52)$ & $2989(0,47)$ \\
\hline 1997 & 19 & $97(9,58)$ & $4210(0,45)$ \\
\hline 1998 & 15 & $172(8,72)$ & $3353(0,45)$ \\
\hline 1999 & 16 & $158(10,12)$ & $3017(0,53)$ \\
\hline 2000 & 18 & $138(13,04)$ & $2857(0,63)$ \\
\hline Total & 96 & $858(11,24)$ & $19082(0,50)$ \\
\hline
\end{tabular}

A regressão logística foi realizada para a percentagem de GE em relação ao número de laparotomias e número de nascidos vivos a cada ano, tentando-se detectar possíveis variações na prevalência de GE no período. A prevalência manteve-se estável nos seis anos estudados (Tabela 4).

\section{Discussão}

A maior parte das mulheres com gestação ectópica era jovem, o que foi compatível com o descrito para a população exposta à patologia, mulheres no período de vida posterior aos primeiros anos de vida sexual ativa, e ainda dentro do período reprodutivo.

Esta amostra contém dois terços de muIheres entre 25 e 35 anos, o que é esperado, entretanto $18 \%$ delas tinham 24 anos ou menos e seis eram adolescentes, o que chama a atenção para mulheres que iniciam atividade sexual precocemente. Além disso, é importante ressaltar que I $5 \%$ delas eram nulíparas, experimentando uma seqüela tubária com provável comprometimento de sua fertilidade futura. Estes dados mostram a importância da patologia para o risco reprodutivo de mulheres jovens e revelam a dificuldade do sistema de saúde na educação e prevenção dessas infecções. A medida preventiva mais importante para prevenção das DST/AIDS ainda é o uso do condom, pouco utilizado entre a população geral ${ }^{10}$. Especialmente entre mulheres adolescentes e jovens, que, apesar de o utilizarem mais em relação às mulheres de faixas etárias mais elevadas, ainda $\mathrm{O}$ utilizam pouco e de forma pouco consistente". Devemos lembrar que as infecções de trato reprodutivo são geralmente mais graves entre as mulheres jovens ${ }^{12}$.
Outra dificuldade é a de diagnosticar precocemente a GE. Um reforço nessa idéia é dado pelo número de cirurgias tubárias mutilantes, que nessa amostra correspondeu a $79 \%$ dos casos. O diagnóstico foi tardio, num momento em que o grau de comprometimento tubário impedia qualquer outra alternativa de tratamento, exceto a retirada da tuba. Chama a atenção o comprometimento ovariano, geralmente tardio nos casos de grandes hematomas, que levou à retirada do anexo em seis mulheres. Esses dados chamam a atenção por serem compatíveis com o descrito por serviços de emergência de países não industrializados que apresentam maiores incidência e complicações de GE. Numa série de 81 muIheres submetidas a cirurgia abdominal de urgência em hospital rural de Sierra Leona, 43 tinham GE rôta e foi descrito um óbito' ${ }^{13}$. Os países desenvolvidos, por outro lado, têm apresentado redução na incidência de gravidez ectópica, como o observado em um distrito londrino no qual houve queda entre os anos de 1990 a 1999 de 2,4 para 1,6 de gestações ectópicas por 100 nascidos vivos ${ }^{14}$.

É correto pensar que as mulheres deste estudo demoraram a ter um diagnóstico, seja porque tardaram a consultar-se, porque foram subestimadas as suas queixas ou porque não tiveram, nos serviços consultados, essa hipótese diagnóstica levantada, ou ainda, se estas foram pensadas, demorou-se na realização de testes acurados de detecção, como o ultrasom. Na rede pública, é mais difíil praticar-se com urgência esse tipo de atenção e é comum que nos casos pouco sintomáticos não haja grande preocupação com a demora do exame, seja pela própria paciente, seja pelo sistema.
O diagnóstico tardio, além de tratamento mais invasivo, tem evolução com maior freqüência de complicações. Encontrou-se 19\% de complicações, sendo a mais freqüente a anemia aguda com necessidade de transfusão ( 12 casos). A norma para este tratamento no serviço, além da indicação por distúrbio hemodinâmico no pré ou intra-operatório é de sintomatologia clínica importante com concomitante dosagem de hemoglobina sérica $\leq 8 \mathrm{~g} \%$ ou hematócrito $\leq 25 \%$ no pós-operatório.

Além disso, ocorreram também complicações intra-operatórias, com três casos de lesão inadvertida de alça, necessidade de traqueostomia por reação a drogas utilizadas e um caso de reintervenção por sangramento no pós-operatório. Ainda, uma mulher evoluiu com quadro grave de choque e CIVD, sem resposta ao tratamento e morte. Esses dados explicitam a alta morbidade provocada pela doença nos dias de hoje, mesmo nas grandes cidades e em serviços de referência.

Nesta série, somente em 20 casos foi realizada cirurgia conservadora, ou seja, com manutenção da tuba após secção longitudinal, aspiração e lavagem do leito de inserção ou pela técnica da ordenha da tuba íntegra, após constatação no intra-operatório de aborto tubário. Não foi objetivo deste estudo avaliar os critérios utilizados para a realização da cirurgia conservadora, entretanto, é de se supor que estas tenham sido as situaç̃̃es em que se teria optado por procedimento laparoscópico e que por algum motivo este não estivesse disponível na ocasião.

Este estudo possui limitação por ser o levantamento de um único centro hospitalar com um número pequeno de mulheres. Entretanto, é de importância ressaltar que o objetivo não foi um estudo de todos os casos da patologia e sim determinar a prevalência de casos submetidos à laparotomia no serviço, casos que não poderiam ser tratados de outra forma, ou seja, com algum tempo de evolução e com um maior comprometimento do estado geral. E esse número não se alterou com o passar dos anos, o que é importante, apesar da necessidade da coleta de dados regionais para medir de forma adequada a dimensão do problema.

Os dados de incidência e prevalência são difíceis de medir em qualquer país. Zane etal. chamaram atenção para o fato de que, 
FERNANDES AMS ET AL.

após tentativas para estimar-se o problema nos EUA buscando dados de hospitalizações, altas cirúrgicas, altas de procedimentos médicos, consultas médicas e casos notificados de $\mathrm{GE}$, encontraram amostras pequenas com cálculos severamente limitados de estimativas de GE e dados incompatíveis quando combinados entre si. Concluíram que o cálculo não poderia ser estimado por não existir em dados confiáveis representativos e que 0 avanço nos métodos diagnósticos e de tratamento haviam afetado a pesquisa de duas formas: o tratamento médico hospitalar da GE havia diminuído e as consultas médicas por GE haviam aumentado ${ }^{15}$.

A aquisição de dados próprios da prevalência de casos de evolução avançada é vital para nortear medidas médicas locais de apoio ao diagnóstico precoce. Entretanto, também é necessário realizar estudos sobre a incidência e prevalência em áreas regionais, com dados recolhidos nos demais serviços. A contribuiç̧ão de estudos mais abrangentes na população será importante para que a sensibilização dos profissionais de saúde e maior atenção ao diagnóstico e tratamento precoces da patologia sejam implementados.

A orientação sobre prevenção das DST dirigida a mulheres e homens deve ser estimulada para diminuir o número de novos casos. Os médicos devem estar atentos ao diagnóstico de clamídia e gonococo, realizar tratamento efetivo e orientar medidas preventivas com o objetivo de diminuir complicações.

A gestação ectópica constitui patologia grave, cursa com alta morbidade e segue sendo desafio constante dentro dos nossos serviços de ginecologia.

\section{Conflito de interesse: não há.}

\section{SUMMARY}

\section{Prevalence of ectopic pregnancy LIABLE TO SURGICAL TREATMENT IN A PUBLIC HOSPITAL FROM 1995 THROUGH 2000}

OBIECTNES. To determine the prevalence of women with ectopic pregnancy submitted to surgical treatment at the HMCP from 1995 to 2000; as well as to evaluate some characteristics of these women and the treatment performed.
METHODS. The study was retrospective. Data was obtained from medical register of women submitted to laparotomy with the diagnosis of ectopic pregnancy, Variables studied were: patient's age, place of birth and occupation, status of ectopic pregnancy (with or without rupture), type of surgery, complications and duration of hospital stay. Data was analyzed in a descriptive way, followed by logistic regression analysis.

REsults. Ninety six cases were studied. Age of the majority of women (65\%) ranged from 25 to 35 years; $6 \%$ were adolescents. Ofall patients $30 \%$ were employed. Distribution according to status of pregnancy: the study showed that $66 \%$ presented rupture; $79 \%$ of the patients were submitted to salpingectomy with or without ooforectomy. Complications: The mostfrequent complication was acute anemia, including one death due to DIVC (disseminated intravascular coagulation), and $12 \%$ of the patients had to receive blood transfusion. The average time of hospital stay was of 3.63 days $(S D \pm 0.81)$. Prevalence of ectopic pregnancies did not change during the period of study and was responsible for $11.2 \%$ of the total number of laparotomies.

CONCLUSIONS. For the period of this study, the number of women submitted to laparotomy due to ectopic pregnancy remained quite high and unchanged For control, this situation requires preventive measures at different levels. Ectopic pregnancy is a severe pathology and induces high rates of morbidity. It remains a challenge for gynecologists. [Rev Assoc Med Bras 2004; 50(4): 4l3-6]

KEY WORDS: Ectopic pregnancy. Hospitalization. Prevalence.

\section{REFERÊNCIAS}

I. Aral SO. Sexually transmitted diseases: magnitude, determinants and consequences. Int J STD AIDS 2001 ; 12:2 I I-5.

2. Nagib $H$, Silva MB. Mortalidade feminina em idade reprodutiva no estado de São Paulo, Brasil, 1991-1995: causas básicas de óbito e mortalidade materna. Rev Saúde Pública 2000; 34:64-70

3. Marchbanks PA, Aneger JF, Coulman. Risk factors for ectopic pregnancy: a population based study. JAMA 1988; 259: 1823-7.
4. Bouyer J, Coste J, Shojaei T, Pouly JL, Fernandez H, Gerbaud L, et al. Risk factors for ectopic pregnancy: a comprehensive analysis based on a large case-control, populationbased study in France. Am J Epidemiol 2003; 157: 185-94.

5. Barlow RE, Cooke ID, Odukoya O, Heatley MK, Jenkins J, Narayansingh $G$, et al. The prevalence of Chlamydia trachomatis in fresh tissue specimens from patients with ectopic pregnancy or tubal factor infertility as determined by PCR and in situ hybridization. J Med Microbiol 200 I; 50:902-8.

6. CDC-Centers for Disease Control. HIV prevention through early detection and treatment of other sexually transmitted diseasesUnited States recommendations of the Advisory Committee for HIV and STD prevention. MMWR Morb Mortal Wkly Rep 1998; 47(RR/2): I-24

7. Ego A, Subtil D, Cosson M, Legoueff F, Houfflin-Debarge V, Querleu D. Survival analysis of fertility after ectopic pregnancy. Fertil Steril 200 I; 75:560-6.

8. Luciano AA, Roy G, Solima E. .Ectopic pregnancy from surgical emergency to medical management. Ann N Y Acad Sci 2001; 943:235-54.

9. Lipscomb GH, McCord ML, Stovall TG, Huff G, Portera SG, Ling FW. Predictors of success of methotrexate treatment in women with tubal ectopic pregnancies. N Engl J Med 1999; 341:1974-8.

10. Pesquisa nacional de demografia e saúde. Rio de Janeiro: BEMFAM; 1996. p.52-8.

I I. Fernandes AMS, Antonio DG, Bahamondes L, Cupertino CV. Conhecimentos, atitudes e práticas de mulheres brasileiras atendidas pela rede básica de saúde com relação às doencas de transmissão sexual. Cad Saúde Pública 2000; I (Supl): $103-12$

12. Igra $\mathrm{V}$. Pelvic inflammatory disease in adolescents. AIDS Patient Care STDS 1998; 12:109-24.

13. McConkey, SJ. Case series of acute abdominal surgery in rural Sierra Leone. World I Surg 2002; 26:509-13.

14. Irvine LM, Setchell ME. Declining incidence of ectopic pregnancy in a UK city health district between 1990 and 1999. Hum Reprod 200 I; 16:2230-4

15. Zane SB, Kieke BA Jr, Kendrick JS, Bruce C. Surveillance in a time of changing health care practices: estimating ectopic pregnancy incidence in the United States. Matern Child Health J 2002; 6:227-36.

Artigo recebido: 27/08/03

Aceito para publicação: 13/02/04 